\title{
Development of a Simplified Smart Agriculture System for Small-scale Greenhouse Farming
}

\author{
Denis Pastory Rubanga, ${ }^{*}$ Katsumori Hatanaka, ${ }^{2}$ and Sawahiko Shimada ${ }^{3}$ \\ ${ }^{1}$ Department of Agricultural Engineering, Graduate School of Agriculture, Tokyo University of Agriculture, \\ Setagaya-ku, Tokyo 156-8502, Japan \\ ${ }^{2}$ Faculty of International Bio-Business, Tokyo University of Agriculture, \\ Setagaya-ku, Tokyo 156-8502, Japan \\ ${ }^{3}$ Faculty of Regional Environmental Science, Tokyo University of Agriculture \\ Setagaya-ku, Tokyo 156-8502, Japan
}

(Received October 12, 2018; accepted January 31, 2019)

Keywords: wireless sensor network, smart agriculture, small-scale greenhouse farmer, microclimate

A challenge exists in horticultural greenhouse farming as a result of the current decline in the agriculture labor force in Japan. Although there is a need for cutting-edge technological Information and Communications Technology (ICT) application in agriculture, few smallscale farmers are willing to risk significant capital on sensing technologies. In this study, a simplified smart agriculture system using limited resource expense was developed and applied in a tomato greenhouse. The system's real-time information capability is composed of commercial inexpensive wireless sensor network (WSN) devices, and a developed web database (web DB) for daily activity data collection was used for crop environment monitoring and management. The crop microclimate environment was monitored on the basis of an agroclimatic index, namely, growing degree day (GDD). Microclimate spatiotemporal distributions using GDD were also determined, which showed well-defined microclimate variations within the greenhouse. Crop calendars were embedded into the developed web DB for tomato farms. We drew conclusions for better crop management and crop yields using our smart agriculture system. The applicability of the system based on initial cost, running costs, and the reliability of WSN data makes it cost-effective. Consequently, it could be used for the accurate crop production planning and decision making of cultivation activities.

\section{Introduction}

The population of Japanese agricultural workers is decreasing, and aging is progressing at the same time. ${ }^{(1)}$ The average age of farmers in Japan is 65.8 years old, and the ratio of workers aged 65 and above to the total agricultural population is $61.8 \%$. Japan is experiencing the arrival of a super-aged society and a full-fledged population-declining society, and thus there is a concern of serious labor shortage in the near future. ${ }^{(1,2)}$ There are efforts to make agriculture a progressive industry. Such efforts include the full utilization of resources and the potential

*Corresponding author: e-mail: 45618006@nodai.ac.jp

https://doi.org/10.18494/SAM.2019.2154 
of rural areas through technological innovation. The initiative of the Ministry of Agriculture, Forestry, and Fisheries (MAFF) of Japan is to promote the introduction of smart agriculture aiming for labor-saving and efficiency improvement using state-of-the-art robot technology and Information and Communications Technology (ICT), ${ }^{(3)}$ and also to undertake research and development initiatives on-site by introducing cutting-edge technologies to realize smart agriculture.

A wireless sensor network (WSN) as a smart agriculture technique was used in this study. WSN devices and various types of optical sensors are assumed to be a basic technology in smart agriculture, which intends to achieve harmony with economic development and sustainable agroecosystem urges. Fitz-Rodríguez et al. ${ }^{(4)}$ and Akyildiz et al. ${ }^{(5)}$ argued that WSN devices have been under rapid development and they have become a promising application in agriculture. Previous studies on smart agriculture have been conducted; for instance, Kameoka and Hashimoto conducted demonstration experiments using WSNs in a mandarin orange orchard and in a vineyard to promote smart cultivation management. ${ }^{(6)}$ Langendoen et al., in their LOFAR-agro project, studied a potato crop resistant to phytophthora (a genus of water mold) by monitoring microclimate conditions (humidity and temperature) using large-scale WSN devices. ${ }^{(7)}$ The system had intentions of generating a policy to protect the crop against fungal disease based on the collected data. Kameoka et al. also developed a new WSN with a weather station and a soil water potential sensor that enabled long-range wireless communication; the data acquired was standardized and used to create a web service that offered various kinds of phenological indices to farmers in the field. ${ }^{(8)}$

In fact, recent technologies in Japan have been initiated by large technological companies based on cloud computing services such as the Fujitsu's ${ }^{(9)}$ Akisai Food and Agriculture Cloud. This service is designed to provide comprehensive support to all aspects of agricultural management, such as administration, production, and sales in the open-field cultivation of rice and vegetables, horticulture, and animal husbandry. Moreover, the Kubota Smart Agricultural System (KSAS) helps visualize agriculture business operations. ${ }^{(10)}$ The various data are collected from rice paddies and uploaded to the cloud server. The data is shared by the farmer and the manager and can be analyzed to yield various types ofinformation. The information can be easily accessed through a PC or a handheld device connected via a wireless local area network (LAN). Data is collected in real time by agricultural machinery via the wireless LAN during operation.

We summarized the $\mathrm{MAFF}^{(11)}$ smart agriculture catalog that contained 150 recognized types of smart agriculture systems. Smart agriculture is subdivided into five categories, i.e., data management (11\%), cultivation management (16\%), environmental management (7\%), automatic and work mitigation (39\%), and sensors for monitoring (28\%). In Japan, smart agriculture application has been realized in farm management, plant growth diagnosis, land preparation, fertilizer application, pesticide application, water management, weed control, harvesting, and so forth. The price of the KSAS basic plan is from 35 USD per month. The cost of the Akisai service is from 400 to 1000 USD per month. In addition to the cost, it is difficult to introduce smart agriculture in small-scale horticultural facilities owing to the lack of knowledge and understanding of the advanced technologies of smart agriculture. Fitz-Rodríguez et al. claimed 
that the fear that farmers cannot handle it even if it is introduced is considered to be a barrier, and the spread is delayed. ${ }^{(9)}$

This study, therefore, was in support of MAFF policies on research efforts to support cutting-edge technological ICT applications in agriculture and also to reduce the cost of expensive smart agriculture systems. The main aim of our research was to establish alternative approaches to ICT development and their applications in horticultural agriculture through the transfer of new advanced technologies to small-scale farmers in a collaborative support from research institutes to improve agriculture production management.

In this study, we developed a simplified smart agriculture system with a real-time information capability that could be used for monitoring crop environment and crop management in a greenhouse at limited resource expense. We used inexpensive WSN devices and collaborated with small-scale strawberry and tomato greenhouse farmers in Tochigi and Nara in Japan, respectively. However, in this paper, we focus on applications in tomato greenhouses.

Real-time microclimate conditions were monitored using WSN devices, and daily activities were collected during crop growth and the data stored in the developed web DB. We used the growing degree day (GDD) technique to monitor crop growth as Nicolosi et al. showed the importance of crop microclimate in crop growth monitoring using the cumulative GDD technique. ${ }^{(12)}$ Also, Miller et al. considered using GDD to monitor crop development, the assumption being that development is limited to a certain threshold. ${ }^{(13)}$ In addition, daily activity data were gathered and stored in a Google Drive spreadsheet as explained in succeeding sections. Crop calendars were also embedded into the web DB for tomato farms (tomato cultivation is about ten crop growth cycles per year). We further showed microclimate spatiotemporal distributions within a tomato greenhouse based on GDD. The conclusions of our study were drawn in terms of how important the developed smart agriculture would be, such as that discussed in this paper, if deployed by small-scale farmers.

\section{Materials and Methods}

\subsection{Smart agriculture system architecture}

The smart agriculture system shown in Fig. 1 was deployed on an already existing tomato greenhouse. The tomato greenhouse uses the soilless cultivation technique called the nutrient film technique (NFT). ${ }^{(14)}$ The system's architecture consisted of physical components for data collection, i.e., WSN devices for microclimate data and a PC for daily activity data. The microclimate data collected were stored in the WSN device cloud accessible by the Internet provided by an installed Wi-Fi router. A PC installed with a Google Drive spreadsheet was accessible to a developed web DB for the input of daily activity data such as fertilizer usage, harvest data, and so on. The collected data were stored on a rental server's database that was also used to host the web DB. WSN devices were distributed at convenient accessibility owing to the limitation of the Wi-Fi signal between the base station and the Wi-Fi router, and between the base station and the connected indoor and outdoor modules. The deployed sensors, greenhouse layout, and database are explained in more detail in the succeeding sections. 


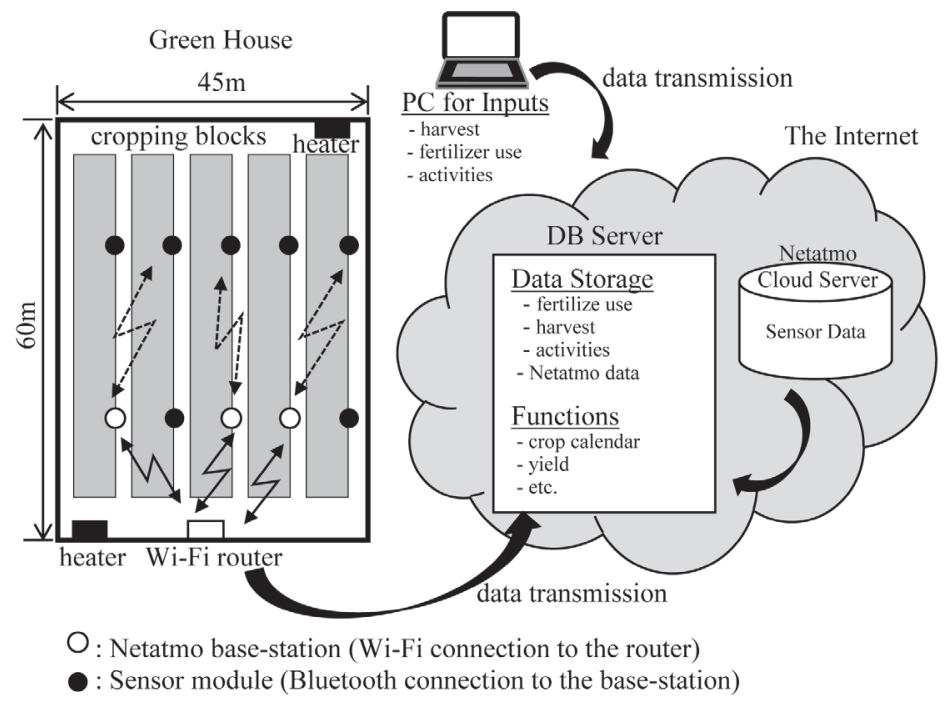

Fig. 1. Illustration of smart agriculture system architecture composed of Netatmo WSN devices for microclimate data collection, a Wi-Fi router for Internet connectivity, a PC for data inputs, and a DB server for data storage and functions, such as a crop calendar and so on.

\subsection{Sensors deployed}

The WSN devices used for the smart agriculture system were purchased from Netatmo. ${ }^{(15)}$ Such devices are equipped with sensors for temperature and humidity, a barometer, a $\mathrm{CO}_{2}$ meter, and a sound meter. See the specifications and measurement range of the sensors in Table 1. They are made of a single piece of durable aluminum shell and are UV-resistant. Netatmo modules are shown in Fig. 2. Two main types of modules were used: indoor and outdoor modules. The indoor modules are of two categories, i.e., base station and additional module. The base station is powered by a USB wall adapter, whereas the outdoor and additional modules are powered by two triple-A batteries (up to 2 years lifespan). A Wi-Fi router (Wi-Fi $802 \mathrm{~b} / \mathrm{g} / \mathrm{n}$ compatible $(2.4 \mathrm{GHz})$ with supported security Open/WEP/WPA/WPA2) with Internet access was required for the sensors to send data to the Netatmo cloud server. The WSN devices are compatible with iOS, Android 4.0 (minimum), and Windows 8.0 (minimum) operating systems. The additional and outdoor modules send their measurements to the base station via Bluetooth. Using the Wi-Fi access point, each indoor module then sends both its own measurements and those of other modules connected to it to a registered Netatmo account. The cost for a set of base station and indoor-outdoor module is 200 USD.

\subsection{Data transmission}

The system data are divided into twocategories, namely, microclimate data collected by the WSN devices and daily activities. The WSN devices collected data at 5 min intervals (for 
Table 1

WSN device specifications.

\begin{tabular}{|c|c|c|c|c|c|c|}
\hline \multirow{2}{*}{ Measurement } & \multicolumn{2}{|c|}{$\begin{array}{c}\text { Temperature } \\
\left({ }^{\circ} \mathrm{C}\right)\end{array}$} & \multicolumn{2}{|c|}{$\begin{array}{c}\text { Humidity } \\
(\%)\end{array}$} & \multirow{2}{*}{$\begin{array}{c}\begin{array}{c}\mathrm{CO}_{2} \text { meter } \\
(\mathrm{ppm})\end{array} \\
\text { Outdoor }\end{array}$} & \multirow{2}{*}{$\begin{array}{c}\begin{array}{c}\text { Barometer } \\
\text { (Mbar) }\end{array} \\
\text { Indoor }\end{array}$} \\
\hline & Indoor & Outdoor & Indoor & Outdoor & & \\
\hline Range & 0 to 50 & -40 to 65 & 0 to 100 & 0 to 100 & 0 to 5000 & 260 to 1260 \\
\hline
\end{tabular}

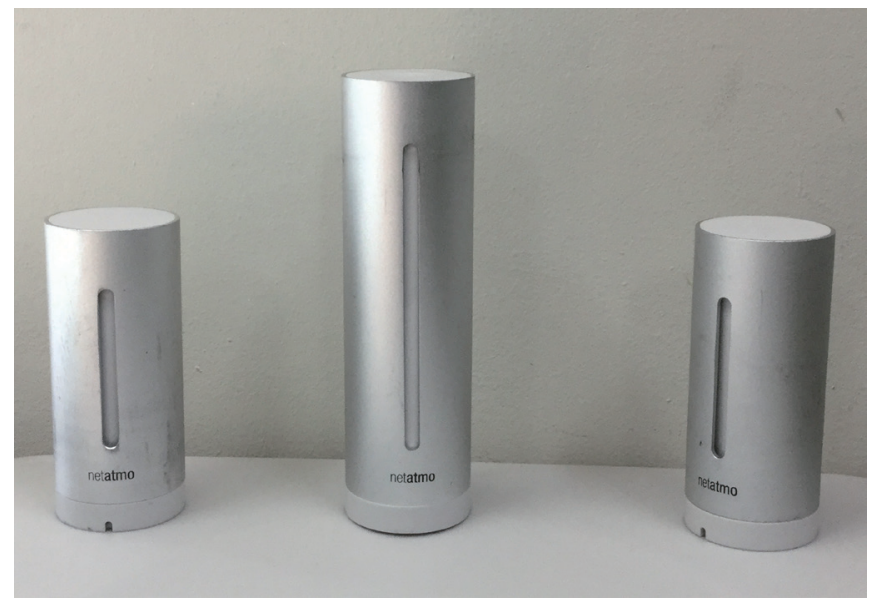

Fig. 2. (Color online) Netatmo sensors used: (left to right) outdoor module, indoor module (base station), and indoor module (additional module).

analysis, we used 30-min-interval data). Daily activities consisted of daily harvest data such as fertilizer usage data [calcium and nitrate solutions, electrical conductivity (EC) concentrations, $\mathrm{pH}$ ] collected by the developed web DB. Planting and harvest duration dates were collected and input manually using a PC installed with a Google Drive spreadsheet. The data input was managed by a greenhouse employee who was ICT-literate. The Wi-Fi router costs 70 USD and the monthly running cost for $1 \mathrm{~GB}$ is $4 \mathrm{USD}$. $0.3 \mathrm{MB}$ of data is transmitted daily per module; thus, $0.9 \mathrm{MB}$ per month for each module. A total of $90 \mathrm{MB}$ of data is transmitted from the 10 WSN devices per month.

\subsection{Data storage}

In our proposed smart agriculture system, as mentioned earlier, daily activity data were collected using a PC. A web DB shown in Fig. 3(a) hosted on a rental server was developed for the entry of daily activity data such as planting and harvest dates, number of transplanted seedlings, and so on. The server composed of a rational MySQL database is used for the storage of data from the Google Drive spreadsheet, daily activity data, and microclimate data from the Netatmo cloud server. By using data of planting and harvesting dates of individual greenhouse blocks, which are stored in the database, a crop calendar [Fig. 3(b)] was created automatically and embedded into the web DB. 


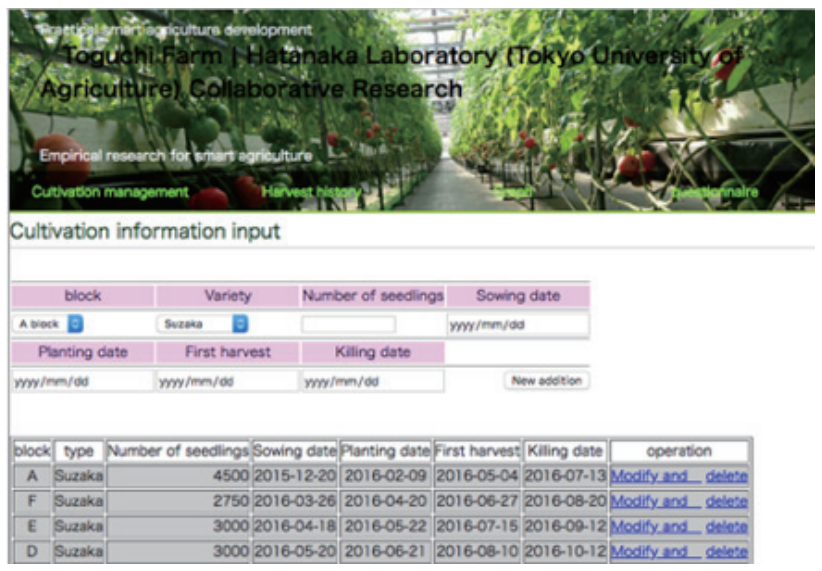

(a)

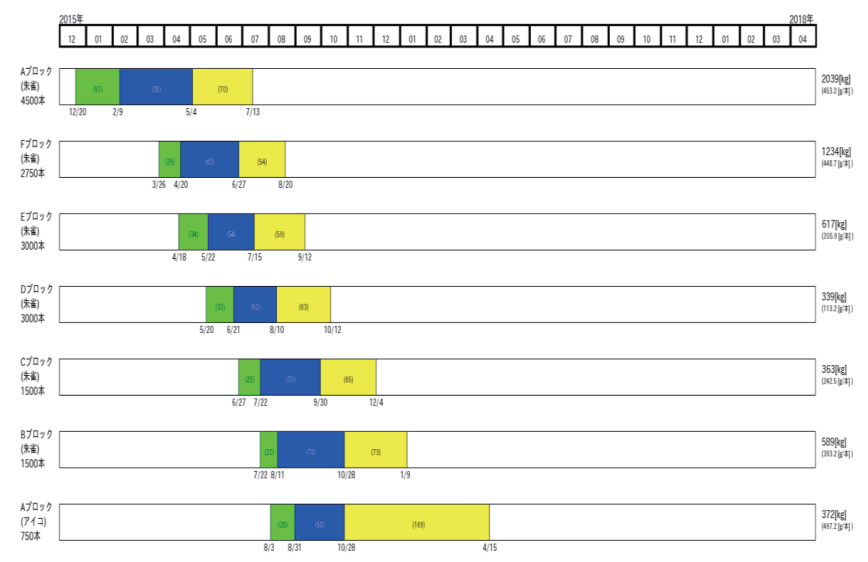

(b)

Fig. 3. (Color) Web DB (a) for daily activity data entry and (b) crop calendar. The green, blue, and yellow rectangles [nursery (green), vegetative growth period (blue), and harvest period (yellow)] represent individual blocks. Each block shows the number of planted seedlings, e.g., 4500 on the left side of the block, and start and finish dates of activities. Values of tomato harvested and yield (right of block). Numbers within each colored rectangle show duration in days. N.B. Japanese characters are used in (b) since it was designed for Japanese farmers.

\section{Deployment in Tomato Greenhouse}

The smart agriculture system was used on a tomato greenhouse of six blocks as shown in Fig. 4(c) with an area of $2500 \mathrm{~m}^{2}$, an angle roof $3.5 \mathrm{~m} \mathrm{high,} \mathrm{and} \mathrm{an} \mathrm{internal} \mathrm{sheeting} \mathrm{of} 3 \mathrm{~m}$ high. Tomato production is carried out in a rotation cycle within one greenhouse. By using NFT, a very shallow stream of water containing all the dissolved nutrients required for tomato growth is recirculated past the roots of the plants in each row in soilless beds (a double row of $40 \mathrm{~cm}$ width, $40 \mathrm{~cm}$ above the floor, and $80 \mathrm{~cm}$ apart for each double row). The supplied nutrient solution consists of calcium $\left(\mathrm{CaCl}_{2} / \mathrm{MgSO}_{4}\right)$ and nitrate solutions. The nutrient solution levels are controlled by determining their $\mathrm{EC}$ and $\mathrm{pH}$. Tomato seedlings are grown in a separate nursery chamber before being transplanted to the greenhouse. Blocks of the same size are F, $\mathrm{E}$, and $\mathrm{D}$, and $\mathrm{A}, \mathrm{B}$, and $\mathrm{C}$. WSN devices were distributed in the tomato greenhouse as shown in the greenhouse layout [Fig. 4(c)]. The WSN devices were installed $2 \mathrm{~m}$ above the floor as shown in Fig. 4(a) to ensure that the microclimate near the tomato plants is measured. The tomato plants are supported by stakes to about $2.5 \mathrm{~m}$ high as shown in Fig. 4(b). In this paper, microclimate parameters between July 2017 and December 2018 were considered for analysis. Blocks A, B, and C shared two WSN devices installed at their boundaries since they were relatively smaller than blocks $\mathrm{D}, \mathrm{E}$, and $\mathrm{F}$.

\subsection{Greenhouse microclimate monitoring approach}

The monitoring of crop growth development was carried out within greenhouse blocks for different complete crop cycles. Snyder et al. argued that the heat unit in GDD is often 


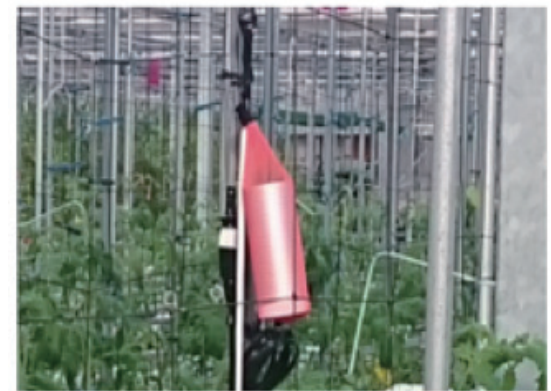

(a)

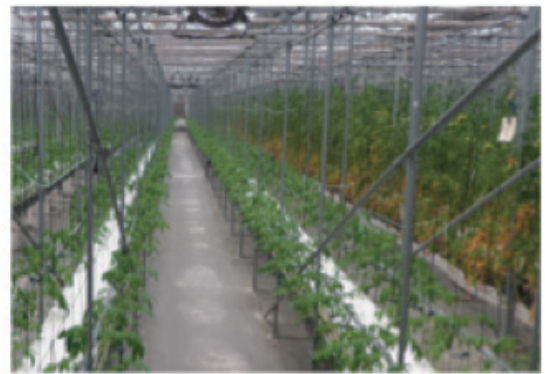

(b)

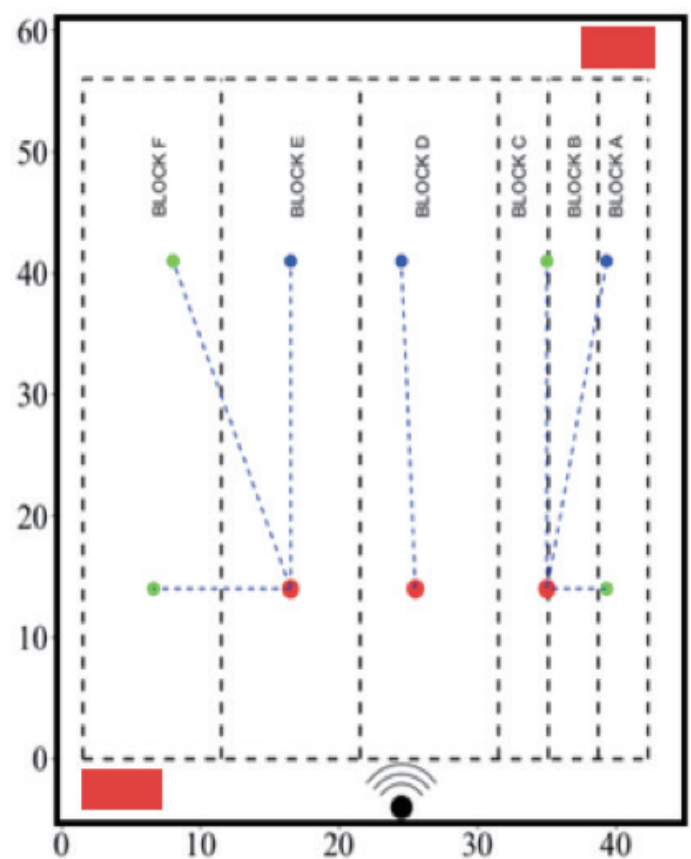

(c)

Fig. 4. (Color) Photograph of (a) WSN device in greenhouse, (b) block within greenhouse, and (c) greenhouse layout showing the location of WSN devices. The blue, green, and red points are the WSN devices. Red points are base station modules that receive data from the additional modules; green points are outdoor modules (blue points) connected via Bluetooth (blue dashed lines) to their respective base stations. Black dashed lines are block boundaries. Black solid lines show greenhouse boundaries. Demarcated values are the distances in meters of length and width. The black point with three curved lines represents the installed Wi-Fi router. Red boxes in (c) stand for heating facilities.

used to predict the phenological development rate of plant species. ${ }^{(16)}$ The phenological developmental rate increases approximately linearly as a function of air temperature, and heat units are a measure of the duration at various temperatures. Therefore, GDD is used to quantify phenological development. However, the development rate is assumed to be insignificant when the air temperature is below the threshold, termed as the base temperature. If the temperature is more than the base temperature, then GDD is equal to the observed air temperature minus the base temperature, as shown in Eq. (1). Therefore, a larger difference between the air temperature and the base temperature implies a larger GDD and a higher development rate. The air temperature variables used were transformed to GDD using the following equation:

$$
G D D=T_{\text {mean }}-T_{C},
$$

where $T_{\text {mean }}$ is the mean daily air temperature (average of daily maximum and minimum temperatures), $T_{C}$ is the threshold or base temperature, and $10{ }^{\circ} \mathrm{C}$ was used as the base temperature. ${ }^{(16)}$ Whenever $T_{\text {mean }}$ is less than the threshold $\left(T_{\text {mean }}<T_{C}\right)$, GDD is set equal to zero $(G D D=0)$. The accumulated GDD within each respective block is used to monitor the 
vegetative growth and harvest periods. For each block, the average GDD for two WSN devices was used. The accumulated GDD was then used in the real-time monitoring of crop growth stages from the time tomato seedlings were transplanted to the end of the crop cycle, i.e., the last harvest date. By using manually collected crop harvest data using a PC and stored in a web DB, the crop yield for each complete crop cycle was determined by the dividing the total daily harvest from each block by the total number of plants. The accumulated crop yield was also determined by the summation of the daily crop yields.

\subsection{Greenhouse spatiotemporal distribution approach}

The spatiotemporal distribution of the microclimate parameters in the greenhouse was determined by WSN device data interpolation. Assuming that microclimate variables are irregularly distributed in the greenhouse, we selected a linear method, namely, a bivariate interpolation method and a smooth surface fitting for values that are given at irregularly distributed points using the Akima method. ${ }^{(17)}$ The linear method is based on a twodimensional Cartesian coordinate system with the length and width of the greenhouse layout considered as the $x-y$ plane and $z$, which represents a smooth surface of the $z$ values at selected points irregularly distributed in the $x-y$ plane. The selected points were the locations of the WSN. All the calculations were carried out using a statistical package R software program installed with the Akima package. Results of the interpolation are discussed in Sect. 4.2.

\section{Results and Discussion}

\subsection{Crop growth monitoring using microclimate}

Crop growth monitoring based on GDD was carried out. Bauer et al. claimed that temperature and other factors such as photoperiod are important factors that affect the transition from vegetative to floral growth. ${ }^{(18)}$ In fact, Ito also evaluated the effect of air temperature on the plant morphological development of other crops. ${ }^{(19)}$ Therefore, for each block in a tomato greenhouse, the corresponding accumulated GDD was determined during the vegetative growth and harvest periods. Tables 2 and 3 show a summary of the accumulated GDD of eight selected blocks during the vegetative growth and harvest periods. It can be seen that the accumulated GDD was different for both vegetative and harvesting periods of each block.

To evaluate the effects of microclimate parameters on tomato crop growth, we determined the crop yield for eight selected complete crop cycles by dividing from the total daily harvest each block by the total number of plants in the block. We further determined the cumulative crop yield by the summation of the daily crop yields. We also determined the daily means of carbon dioxide and humidity of each block using the average means of each specific WSN device.

From Fig. 5, we see that GDD differs throughout the year. GDD is seemingly high in July and September and low between November and February. The different microclimate conditions in the greenhouse as shown by the GDD graph could imply that tomato plant growth 
Table 2

Summary of accumulated GDD for vegetative growth for eight complete tomato growth cycles.

\begin{tabular}{lccc}
\hline \multirow{2}{*}{ Block } & \multicolumn{3}{c}{ Vegetative growth } \\
\cline { 2 - 4 } & Transplanted & Just before harvest & Acc.GDD* \\
\hline B & $2017-08-21$ & $2017-09-29$ & 624.4 \\
B & $2017-08-21$ & $2017-10-13$ & 849.6 \\
C & $2017-07-18$ & $2017-09-18$ & 1187.0 \\
C & $2017-12-24$ & $2018-04-09$ & 875.9 \\
D & $2017-10-26$ & $2018-03-05$ & 1046.4 \\
D & $2017-05-26$ & $2017-07-25$ & 1030.5 \\
E & $2017-10-05$ & $2018-02-25$ & 1462.6 \\
F & $2017-08-16$ & $2017-10-23$ & 1121.0 \\
\hline
\end{tabular}

*Acc.GDD: accumulated GDD.

Table 3

Summary of crop yield and accumulated GDD for harvest periods for eight complete tomato growth cycles.

\begin{tabular}{lcccccc}
\hline \multirow{2}{*}{ Block } & \multicolumn{5}{c}{ Harvest period } \\
\cline { 2 - 7 } & Harvest start & Harvest end & Acc.GDD & Duration (d) & Total harvest (kg) & Crop yield SD \\
\hline B & $2017-09-29$ & $2018-03-19$ & 1518.0 & 58 & 298.0 & 3.65 \\
B & $2017-10-13$ & $2018-03-19$ & 1288.5 & 56 & 220.2 & 3.85 \\
C & $2017-09-18$ & $2017-12-13$ & 890.3 & 38 & 576.0 & 5.59 \\
C & $2018-04-09$ & $2018-07-23$ & 1563.4 & 47 & 736.8 & 6.76 \\
D & $2018-03-05$ & $2018-07-03$ & 1460.2 & 52 & 1615.6 & 10.83 \\
D & $2017-07-25$ & $2017-10-16$ & 1194.5 & 37 & 1147.5 & 16.06 \\
E & $2018-02-25$ & $2018-05-07$ & 769.6 & 29 & 1214.9 & 12.04 \\
F & $2017-10-23$ & $2018-02-23$ & 1383.4 & 46 & 1398.3 & 7.08 \\
\hline
\end{tabular}

(a)

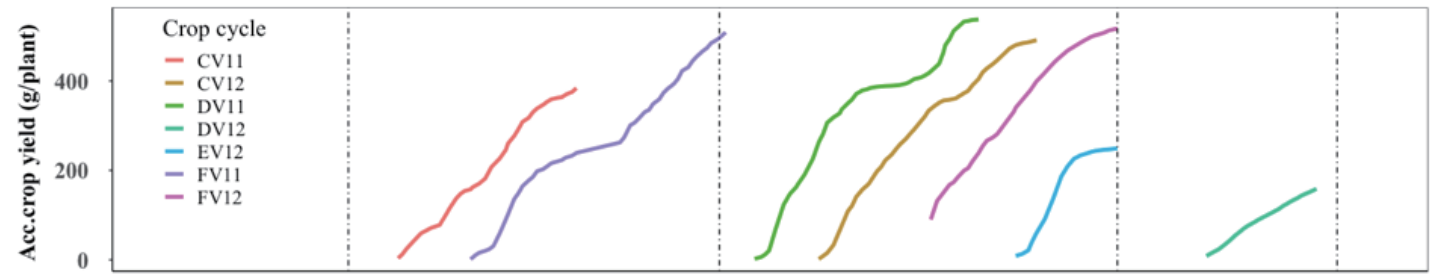

(b)

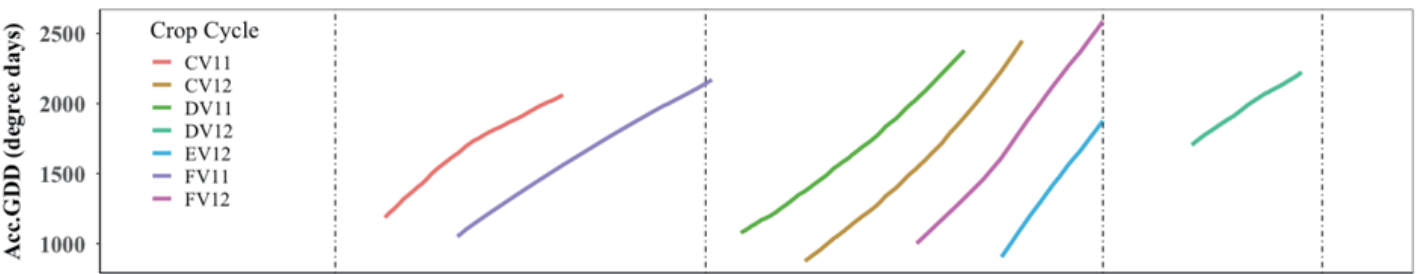

(c)

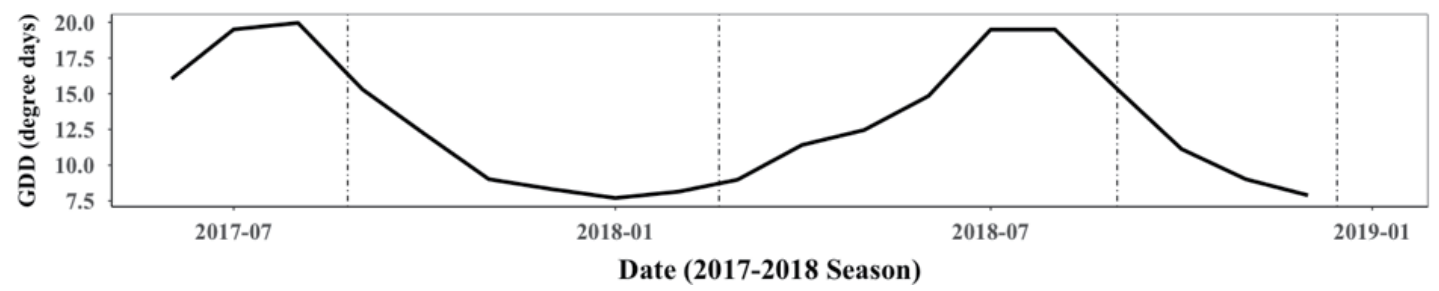

Fig. 5. (Color) (a) Cumulative crop yield of selected crop cycle. (b) Accumulated GDD of selected crop cycle. (c) Daily GDD within the greenhouse. Acc.GDD denotes accumulated GDD and Acc.crop yield denotes accumulated daily crop yield. Crop cycle from CV11 to FV12, where the first letters (C, D, E, and F) denote block names, and appended V11 and V12 denote the first and second crop cycles of a normal crop variety, respectively. 
is affected by microclimate. Accumulated crop yield sigmoid curves of the selected crop cycle were different as well throughout the year.

To determine the response of tomato growth to microclimate conditions and nutrient parameters, the analysis of variance of microclimate parameters and nutrient solution was carried out. The results indicated that all the parameters were significantly different $(p<0.001)$ for nitrate and calcium solutions and EC. Also, GDD, mean humidity, and mean carbon dioxide concentration were significantly different $(p<0.001)$. For the selected complete crop cycles, a significant difference $(p<0.05)$ was observed for crop yield. This could suggest that crop yield differed among all the blocks. We further determined the linear relationships among microclimate, nutrient solution, EC, and tomato crop yield as summarized in Table 4.

From Table 4, models 1, 2, and 3 are linear regression models using the cumulative crop yield as the dependent variable and the 4-day daily moving averages of (b) humidity, (c) carbon dioxide, (d) nitrate solution, (e) calcium solution, and (f) EC as the independent variables. Model 3 is a multiple linear regression model using the accumulated GDD (denoted as a) as the independent variable and the 4-day daily moving average of carbon dioxide. Model 4 is a linear regression model using the accumulated GDD as the independent variable.

Results of the analysis indicated a strong linear relationship $\left(r^{2}>0.89\right.$; models 2 , 3, and 4) between the accumulated GDD and the carbon dioxide and cumulative crop yield for all the seven selected crop cycles. The results of four crop cycles indicated a linear relationship among

Table 4

Summary of crop yield and accumulated GDD for harvest periods for seven complete tomato growth cycles.

\begin{tabular}{|c|c|c|c|c|c|c|c|c|}
\hline Model & & CV11 & CV12 & DV11 & DV12 & EV12 & FV11 & FV12 \\
\hline \multirow{3}{*}{ Model $4^{\mathrm{a}}$} & $r^{2}$ & 0.974 & 0.923 & 0.894 & 0.995 & 0.897 & 0.970 & 0.965 \\
\hline & $p$-value & $* * *$ & $* * *$ & $* * *$ & $* * *$ & $* * *$ & $* * *$ & $* * *$ \\
\hline & AIC & 247.6 & 268.0 & 363.4 & 117.8 & 170.9 & 232.5 & 238.9 \\
\hline \multirow{3}{*}{ Model $3^{\mathrm{a}, \mathrm{c}}$} & $r^{2}$ & 0.982 & 0.993 & 0.978 & 0.996 & 0.931 & 0.973 & 0.996 \\
\hline & $p$-value & $* * *$ & $* * *$ & $* * *$ & $* * *$ & $* * *$ & $* * *$ & $* * *$ \\
\hline & AIC & 240.2 & 213.5 & 321.5 & 117.0 & 167.2 & 232.4 & 195.3 \\
\hline \multirow{3}{*}{ Model $2^{\mathrm{a}}$} & $r^{2}$ & 0.974 & 0.923 & 0.894 & 0.995 & 0.897 & 0.970 & 0.965 \\
\hline & $p$-value & $* * *$ & $* * *$ & $* * *$ & $* * *$ & $* * *$ & $* * *$ & $* * *$ \\
\hline & AIC & 227.3 & 248.5 & 301.8 & 84.8 & 140.5 & 192.1 & 201.6 \\
\hline \multirow{3}{*}{ Model $1^{\mathrm{d}}$} & $r^{2}$ & & 0.520 & 0.008 & 0.872 & 0.584 & 0.267 & 0.869 \\
\hline & $p$-value & & $* * *$ & 0.56 & $* * *$ & $* * *$ & $* * *$ & $* * *$ \\
\hline & AIC & & 456.8 & 586.2 & 201.6 & 225.8 & 584.3 & 346.0 \\
\hline \multirow{3}{*}{ Model $1^{\mathrm{f}}$} & $r^{2}$ & & 0.861 & 0.107 & 0.893 & 0.884 & 0.867 & 0.597 \\
\hline & $p$-value & & $* * *$ & 0.028 & $* * *$ & $* * *$ & $* * *$ & $* * *$ \\
\hline & AIC & & 409.7 & 581.5 & 197.5 & 200.3 & 505.7 & 382.0 \\
\hline \multirow{3}{*}{ Model $1^{\mathrm{e}}$} & $r^{2}$ & & 0.520 & 0.934 & 0.872 & 0.584 & 0.463 & 0.869 \\
\hline & $p$-value & & $* * *$ & $* * *$ & $* * *$ & $* * *$ & $* * *$ & $* * *$ \\
\hline & AIC & & 456.8 & 464.3 & 201.6 & 225.8 & 570.0 & 346.0 \\
\hline \multirow{3}{*}{ Model $1^{\mathrm{b}}$} & $r^{2}$ & & 0.273 & 0.168 & 0.237 & 0.218 & 0.209 & 0.236 \\
\hline & $p$-value & & $* * *$ & $* *$ & 0.018 & 0.038 & $* * *$ & $* *$ \\
\hline & AIC & & 472.6 & 578.3 & 242.6 & 238.5 & 587.8 & 402.5 \\
\hline
\end{tabular}

*Significant at $p<0.05 ; * *$ significant at $p<0.005 ; * * *$ significant at $p<0.001$. 
nitrate solution, calcium solution, EC, and crop yield. There was no observed linear relationship between humidity and crop yield. Results of the linear relationship between microclimate and crop yield could indicate that tomato growth is affected by microclimate.

On the basis of the hypothesis that the microclimate within the greenhouse is not equally distributed, we determined the microclimate spatiotemporal distribution using only temperature parameters as discussed in Sect. 4.2.

\subsection{Spatiotemporal distribution}

To understand the factors for varying the tomato crop yield, it was important to determine the spatiotemporal microclimate distribution in the greenhouse. Prior to determining the spatiotemporal distribution of GDD, the analysis of variance of WSN device parameters was carried out. Results showed significance differences $(p<0.001)$ for temperature and humidity. There were significant differences $(p<0.001)$ in carbon dioxide among the WSN devices (only three WSN devices had a significant difference with a $p$ value of more than 0.05). Results showed that there were significant spatial and temporal variations within the greenhouse especially in the winter season. As shown in Fig. 6, the spatiotemporal distributions of monthly averages of GDD in November and January have distinct variations, and this clearly shows how microclimate conditions within the greenhouse could be different in the winter season.

From the spatiotemporal variation graphs, it is observed that the hotter area at specific spatial locations in the greenhouse depicted the location of the heating facility (heating facility is located in the two corners of the greenhouse).

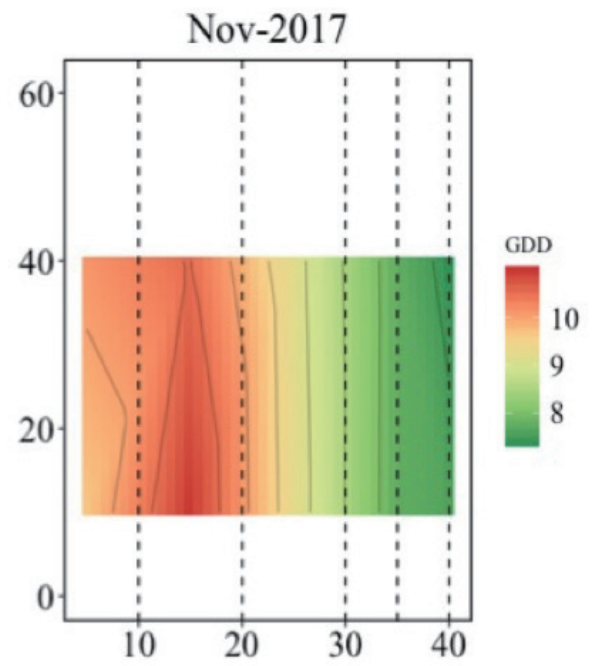

(a)

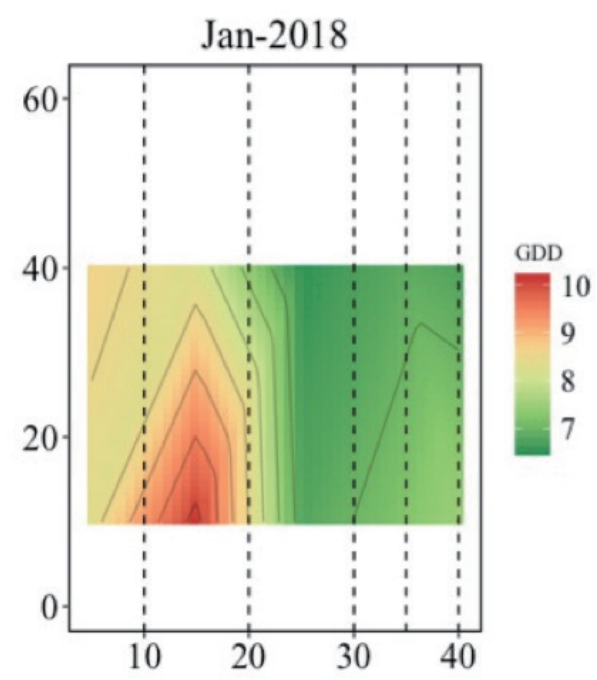

(b)

Fig. 6. (Color) Spatiotemporal monthly GDD variations in tomato greenhouse for November 2017 and January 2018. Values on vertical and horizontal axes represent the greenhouse length and width in meters, respectively. 


\section{Conclusions}

In this study on the development of a simplified smart agriculture system for small-scale greenhouse farming, we draw conclusions on how important smart agriculture could be if used effectively with a deepened understanding of smart agriculture application in smallscale horticultural farming in the case of tomato production. We discussed the application of the smart agriculture system with a real-time capability and for reliable monitoring within the greenhouse. We should note that tomato production is carried out in a rotation cycle within one greenhouse. This study shows the necessity of monitoring specific block microclimate conditions in real time. We were able to extract knowledge from the WSN device data in order to make intelligent decisions to help in farm decision making.

We also selected a simple algorithm such as GDD and showed how it could be used for crop growth monitoring. The use of only temperature in a simplified algorithm is a dimensionality reduction of many parameters collected by WSN devices. We used only GDD data in determining the spatiotemporal variations in the greenhouse and showed how important it was to maintain a well-balanced microclimate environment in the greenhouse. Therefore, to ensure a well-controlled microclimate within the greenhouse, farmers should regulate heating and cooling facilities for an optimum growing environment during the plant growth stage, leading to a reduction in the energy required for greenhouse heating and cooling.

As stated earlier, the challenge of the shortage of agricultural labor would therefore imply using such a simplified smart agricultural system in a labor-demanding situation. Taking into account the initial and running costs as mentioned in Sects. 2.2 and 2.3, the developed system is cost-effective relative to functionality in a limited-resource small-scale horticultural farm. The reliability of the WSN device data makes it efficient, and consequently, it could be used for the accurate crop production planning and decision making of cultivation activities. This was achieved by using the crop calendar that was integrated in the web DB. The crop calendar helps farmers visualize farm activities and therefore reschedule farm activity to enhance efficient farm management. The results of this research imply that, by using a simplified smart agriculture system, the application could be transferred to other tomato greenhouses.

\section{Acknowledgments}

This research was supported by the Tokyo University of Agriculture Research Institute. The authors also thank Mr. Yoshimi Higashiguchi and Mr. Seiichi Higashiguchi of Toguchi Farm for their kind help and fruitful discussion toward this study.

\section{References}

1 T. Kameoka and A. Hashimoto: IEICE Trans. Commun. 98 (2015) 1741.

2 G. Nicolosi, R. Volpe, and A. Messineo: Energies 10 (2017) 722.

3 M. C. Singh, A. Yousuf, and J. P. Singh: Res. Environ. Life Sci. 9 (2016) 1552.

4 E. Fitz-Rodríguez, C. Kubota, G. A. Giacomelli, M. E. Tignor, S. B. Wilson, and M. McMahon: Comput. Electron. Agric. 70 (2010) 105.

5 I. F. Akyildiz, W. Su, Y. Sankarasubramaniam, and E. Cayirci: Comput. Networks 38 (2002) 393. 
6 T. Kameoka and A. Hashimoto: Wireless Sensor Networks and Ecological Monitoring, Vol. 3, eds. S. C. Mukhopadhyay and J.-A. Jiang (Springer, Berlin, 2013) pp. 217-246.

7 K. Langendoen, A. Baggio, and O. Visser: Proc. 20th IEEE International Parallel \& Distributed Processing Symposium (IEEE, 2006) 1530. https://doi.org/10.1109/IPDPS.2006.1639412

8 S. Kameoka, S. Isoda, A. Hahimoto, R. Ito, S. Wada, N. Watanabe, T. Yamakami, K. Suzuki, and T. Kameoka: Sensors 17 (2017) 966.

9 Fujitsu (2016): www.fujitsu.com (accessed July 20, 2016).

10 Kubota Report: www.kubota-global.net/report (accessed July 20, 2016).

11 MAFF (Ministry of Agriculture Fishery and Forestry, Japan, 2018): http://www.maff.go.jp/j/kanbo/kihyo03/ gityo/smart_agri_technology/attach/pdf/smartagri_catalog-3.pdf

12 G. Nicolosi, R. Volpe, and A. Messineo: Energies 10 (2017) 722.

13 P. Miller, W. Lanier, and S. Brandt: Using Growing Degree Days to Predict Plant Stages (Ag/Extension Communications Coordinator, Communications Services, Montana State University-Bozeman, Bozeman, 2001): http://landresources.montana.edu/soilfertility/documents/PDF/pub/GDDPlantStagesMT200103AG.pdf

14 H. Zekki, L. Gauthier, and A. Gosselin: J. Am. Soc. Hort. Sci. 6 (1996) 1082.

15 Netatmo: Netatmo Website: www.netatmo.com (accessed May 20, 2016).

16 R. L. Snyder, D. Spano, C. Cesaraccio, and P. Duce: Int. J. Biometeorol. 42 (1999) 177.

17 H. Akima: ACM Trans. Math. Software (TOMS) 4 (1978) 148.

18 A. Baur, A. B. Frank, and A. L. Black: Agron. J. 76 (1984) 829.

19 H. Ito and T. Saito: Tohoku J. Agric. Res. (1962) 191.

\section{About the Authors}

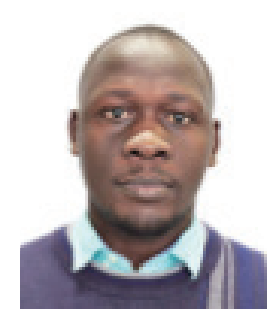

Denis Pastory Rubanga received his B.S and M.S. Agribusiness Management degrees from Tokyo University of Agriculture, Japan, in 2016 and 2018, respectively. Currently, he is a Ph.D. Agricultural Engineering candidate at the Graduate School of Agriculture, Tokyo University of Agriculture. His research interests are applied data science in agriculture and agroinformatics. (45618006@nodai.ac.jp)

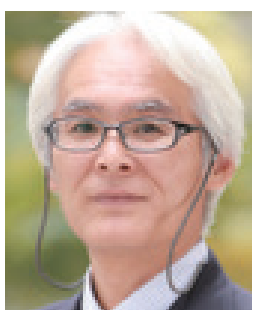

Katsumori Hatanaka received his M.S. and Ph.D. Civil Engineering degrees from Chuo University, Graduate School of Science and Engineering Institute of Technology, Japan, in 1991 and 1993, respectively. From 2007 to 2012, he was an associate professor, and from 2012, he has been a professor at Tokyo University of Agriculture, Japan. His research interests are agricultural environmental engineering and agricultural information.

(k3hatana@nodai.ac.jp)

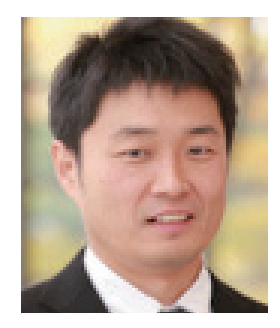

Sawahiko Shimada received his B.S., M.S., and Ph.D. degrees from Hokkaido University, Japan, in 1993, 1996, and 2001, respectively. He joined Tokyo University of Agriculture in 2002. From 2009 to 2010, he was a visiting scholar at Montclair State University, New Jersey, USA. Since 2014, he has been a professor. His research interests are in environmental dynamics analysis, evaluation of carbon accumulation environment in tropical peat swamp forests, and monitoring of the recovery of peat swamp forests using satellite remote sensing data. (shima123@nodai.ac.jp) 